\title{
Quaderni
}

QUADERNI Communication, technologies, pouvoir

74 | Hiver 2010-2011

Récit et information télévisée

\section{La forme du récit dans l'information télévisée}

Avant-propos

\section{Benjamin Berut}

\section{CpenEdition}

Journals

Édition électronique

URL : http://journals.openedition.org/quaderni/334

DOI : 10.4000/quaderni.334

ISSN : 2105-2956

Éditeur

Les éditions de la Maison des sciences de l'Homme

Édition imprimée

Date de publication : 15 janvier 2011

Pagination : 5-8

Référence électronique

Benjamin Berut, «La forme du récit dans l'information télévisée », Quaderni [En ligne], 74 | Hiver

2010-2011, mis en ligne le 05 janvier 2013, consulté le 23 septembre 2020. URL : http://

journals.openedition.org/quaderni/334 ; DOI : https://doi.org/10.4000/quaderni.334 


\section{$D$ ossier}

\section{la forme \\ du récit dans \\ l'information \\ télévisée}

\section{Benjamin Berut}

Responsable du numéro "Récit et information télévisée"

Docteur en Sciences Politiques
Post-doctorant au Céditec
On sait depuis longtemps que le récit est l'une des formes premières de la connaissance, qu'il est un moyen pour l'humain d'appréhender le monde, de le comprendre et d'échanger avec les autres. C'est pour cela que pour Barthes, il n'y a jamais eu de société sans récit ${ }^{1}$. Le récit est alors un objet qui doit forcément intéresser tous ceux qui veulent se pencher sur les moyens qu'a une société de se connaître elle-même, de faire corps, puisqu'il semble bien être l'un des ciments fondamentaux capable d'assurer la cohésion et l'existence d'un groupe. En fait, là où il y a récit il y a société, et où il y a société il y a récit.

Les questions qui se posent sont nombreuses : de quoi parlent ces narrations qui construisent les groupes ? Uniquement d'évènements fictifs ou aussi d'évènements factuels ? Et si on les retrouve dans toutes les sociétés, comment s'y incarnent-elles ? Bref, que racontent-elles, comment le racontent-elles et pourquoi sont-elles capables de construire des groupes, des sociétés entières qui se retrouvent dans le monde qu'elles décrivent ? Alors, on peut poser ici la question la plus simple : quel intérêt pour un numéro de Quaderni de s'arrêter sur la forme du récit dans l'information?

Tout d'abord parce que le récit est à la mode, et la recherche se trouve régulièrement dans une position de critique des concepts trop simples que peuvent répéter à l'envi les journalistes, éditorialistes et essayistes. C'est le cas du storytelling, une notion vague, finalement plus politicienne que politique, lancée Christian Salmon ${ }^{2}$ et massivement reprise, au moins pour un temps qui aura suffi à lui faire intégrer l'imaginaire collectif comme un moyen de propagande quasi 
parfait. Voilà sans doute l'objectif premier de ce numéro : refaire un point théorique de fond sur l'usage de la notion de récit pour comprendre les médias d'information en général et l'actualité à la télévision en particulier. Car c'est bien d'un angle d'attaque dont il s'agit de définir les contours et l'apport aussi bien pour les sciences de la communication que pour la science politique et ce qui apparaît comme l'anthropologie des médias. En effet, considérer l'information comme un récit n'est plus l'oxymore programmatique dont Marc Lits expliquait encore il y a 13 ans que «l'enjeu consiste bien à vérifier s'il n'y a pas un paradoxe, voire une erreur méthodologique ou conceptuelle, à appliquer aux séquences, entre autres informationnelles, l'étiquette de "récit", laquelle suppose construction narrative, mise en intrigue, là où il n'y a peut-être que relation de faits, mais aussi argumentation. $»^{3}$. Aujourd'hui il est acquis que l'information peut être analysée sous la forme du récit. Mais il faut bien sûr aller plus loin, et c'est justement ce que propose ce numéro de Quaderni : poser les enjeux théoriques d'une telle approche et ensuite ses apports à l'étude et à la compréhension du rôle que jouent les mass media dans la construction des identités et des agir collectifs qui peuvent réunir aussi bien un petit groupe qu'un pays dans son ensemble.

Car la narration est une monstration, ainsi pour D. Dayan, les images de la télévision « appellent une analyse qui est moins celle que l'on consacrerait à un tableau que celle que l'on consacrerait à un tableau dans un musée. $»^{4}$. Ce n'est pas tant la peinture, la sculpture ou la pièce ancienne qui est importante que le dispositif qui l'entoure pour orienter le regard du visiteur. Nous pouvons ainsi être des milliers par jour à passer et partager le même point de vue sur un même objet. Les études qui font de l'information un récit ne portent donc pas tant sur l'évènement qui est montré que sur la façon dont il est montré et comment celle-ci peut construire des perceptions, voire des engagements collectifs à travers la construction d'un même point de vue. Ce que nous analysons avec un récit d'information, c'est l'ensemble du système de monstration d'un événement : ses origines, ses valeurs, ses moyens de vraisemblance... L'analyse narrative de l'information touche bien sûr les sciences de l'information et de la communication, mais s'élargit aussi très vite aux sciences politiques, voire à l'anthropologie quand il s'agit d'étudier des récits sous-jacents qui nous possèdent plus que nous les possédons et qui peuvent faire le cœur de notre culture collective. Finalement, l'intérêt de l'angle narratif réside dans sa capacité à nous faire concevoir les prises de positions communes, les identités et les agir collectifs en nous donnant à voir la signification du sens que déploient les médias et le rôle qu'ils tiennent dans la société en nous proposant de partager une prise de position visà-vis d'un événement.

C'est là que volent en éclat les théories de Salmon sur le storytelling et toutes celles qui voient dans la narration un moyen de propagande grâce auquel un petit nombre maitriserait les émotions de la population. Les narrations de l'information télévisée ne sont pas un objet sûr et sous contrôle, elles sont bien plus, elles sont l'une des parties émergées du discours dominant dans une société. Raison pour laquelle elles peuvent se confronter autour d'une même image. En proposant de les voir sous l'angle de l'anthropologie dogmatique que propose Pierre 
Legendre $^{5}$, on pourrait dire que la narration dans l'information télévisée est l'un des vecteurs qui portent ces vérités indémontrées et répétées sur lesquelles les sociétés peuvent exister. En effet, à quoi ressemblerait un groupe où il faudrait à chaque discussion, à chaque rencontre ou à chaque acte de langage remettre en cause et rediscuter rationnellement tous les lieux communs évoqués ? Un groupe ne pourrait tout simplement pas exister s'il devait constamment redéfinir ses lieux communs. Nos sociétés ont donc besoin de ces indémontrés récités que charrient les narrations. Toujours entre innovation et répétition, les récits de l'information télévisée donnent donc accès à une compréhension de la monstration d'un événement et ne nous renseignent donc pas tant sur celui-ci que sur l'image qu'une société veut s'en donner pour rester une société, c'està-dire une entité collective, grâce à une prise de position commune.

Ce que propose alors ce numéro de Quaderni, c'est une analyse théorique, appuyée par des études de cas, des possibles de l'analyse de l'information sous l'angle de la narration.

Christine Servais ouvre la réflexion à travers un article qui fait le point sur les différentes théories du récit pour, après avoir mis en avant que le récit apparaît avant tout comme un moyen d'intégrer l'inconnu au connu, proposer de différencier récitation et narration afin de montrer les spécificités du récit d'information.

Marc Lits vient ensuite poursuivre le numéro à travers un article qui démontre les possibles de l'analyse de l'information sous la forme du récit, en élargissant celle-ci aux récits fragmentés que l'on peut rencontrer aujourd'hui et qui demandent de donner toute leur place aussi bien aux codes narratifs et stéréotypes utilisés qu'à la réception que chaque individu peut en avoir.

Au milieu de tous ces codes narratifs qui se répètent pour construire notre identité collective, la question qui se pose est alors celle du mode de fabrication des récits d'information, Olivier Voirol voit dans cette phase deux pôles entre lesquels se trouve un récit : l'exploration qui tente de réunir la pluralité des sens que l'on peut donner à un même événement et l'oblitération qui, à l'inverse, se fonde sur des indémontrés.

Face à un récit d'information, la problématique ce n'est plus alors celle de sa construction, mais des sens qui sont mis en avant ou laissés de côté. L'enjeu devient donc politique dans une société où l'opinion publique se façonne avant tout à travers les grands médias nationaux. Jocelyne Arquembourg-Moreau étudie cette part des narrations de l'information pour démontrer le rôle qu'elles peuvent jouer dans la société.

Les récits d'information apparaissent alors comme des moyens de la mise en place des identités collectives, Benjamin Berut propose donc, à travers le traitement de la guerre d'Irak en 2003, de comprendre comment se construisent ces récits qui peuvent devenir dominants et mobiliser parfois un ou plusieurs pays.

Jennifer Devresse conclut le numéro justement en apportant une réflexion sur l'agir que peuvent engendrer ces récits qui sont avant tout fondés sur l'émotion. Grâce à l'étude de l'affaire Calice, une affaire de pédophilie dans l'Église qui 
touche actuellement la Belgique, elle démontre comment les téléspectateurs sont invités à prendre position vis-à-vis des acteurs de l'évènement à travers les registres de l'émotion. Elle pose alors la question de la possibilité de la réflexion et de l'agir collectif quand ceux-ci partent des codes de l'émotion.
$\mathrm{N} \cdot \mathrm{O} \cdot \mathrm{T} \cdot \mathrm{E} \cdot \mathrm{S}$

1. R. Barthes, Introduction à l'analyse structurale des récits, in L'analyse structurale du récit, Communications 8, 1966, republié aux éditions du Seuil, coll. Points essais, Paris, 1982.

2. Christian Salmon, Storytelling, la machine à fabriquer des histoires et à formater les esprits, Paris, La Découverte, 2007.

3. Marc Lits, «Le récit médiatique : un oxymore programmatique ? », Recherches en communication $\mathrm{n}^{\circ}$ 7, 1997, pp. 37-38.

4. D. Dayan, Quand montrer c'est faire, in La terreur spectacle, Ina De Boeck, Bruxelles, 2006, p. 166.

5. P. Legendre, De la société comme texte. Linéaments d'une anthropologie dogmatique, Fayard, Paris, 2001. 